\title{
A Scaffolding Design Framework for Developing Secure Interoperability Components in Digital Manufacturing Platforms
}

\author{
Francisco Fraile \\ CIGIP \\ Universitat Politècnica de \\ València Valencia, Spain \\ ffraile@cigip.upv.es \\ Eduardo Saiz \\ Operations and \\ Maintenance Technologies \\ IKERLAN \\ Arrasate-Mondragón, Spain \\ esaiz@ikerlan.es
}

\author{
José Luis Flores \\ Dependable Embedded Systems \\ IKERLAN \\ Arrasate-Mondragón, Spain \\ jlflores@ikerlan.es \\ Raúl Poler \\ CIGIP Universitat \\ Politècnica de València \\ Valencia, Spain \\ rpoler@cigip.upv.e
}

\author{
Víctor Anaya \\ CIGIP \\ Universitat Politècnica de València \\ Valencia, Spain \\ vanaya@cigip.upv.es
}

\begin{abstract}
This paper presents the Virtual Open Operating System (vf-OS) Input / Output (IO) Toolkit Generator, which is a design tool to develop vf-OS IO components that interact with all kinds of manufacturing assets, either physical devices like Program Logic Controllers (PLCs), software applications like Enterprise Resource Planning Systems (ERPs) or legacy file formats like STEP. The vf-OS IO Toolkit Generator is based on software scaffolding, a code generation technique that allows a developer to create a working component to interact with a manufacturing asset from the vf-OS Platform without writing a line of code. As described in this paper, software scaffolding not only simplifies the development of interoperability components, but it also fosters system security and platform integration automation. Another contribution of this paper is to propose possible integrations between the IO Toolkit Generator and the vf-OS Security Command Centre in charge of platform security. Additionally, this paper describes how the concept can be extended to address other digital manufacturing platforms like Fi-Ware.
\end{abstract}

Keywords-System Interoperability, Cyber-Physical Systems, Digital Manufacturing Platforms, Connected Smart Factories, Cybersecurity

\section{INTRODUCTION}

The introduction of the Industrial Internet of Things (IIoT), Internet of Services (IoS) and Cyber-Physical Systems (CPS) into the manufacturing environment has the potential to transform the manufacturing industry in such a disruptive way that it is regarded as a new industrial revolution, Industry 4.0 [1]. Companies of all sizes and sectors acknowledge the benefits and importance of this transformation, but Small and Medium Enterprises (SMEs) with limited resources for Research and Development face difficulties to gain access to these technologies [2].

The Virtual Open Operating System (vf-OS) Platform has been specifically designed to bridge this gap for SMEs, by applying the smart phone application development paradigm to the manufacturing and logistics ecosystem [3]. The main stakeholders and interactions with the vf-OS Platform concept is illustrated in Fig. 1.

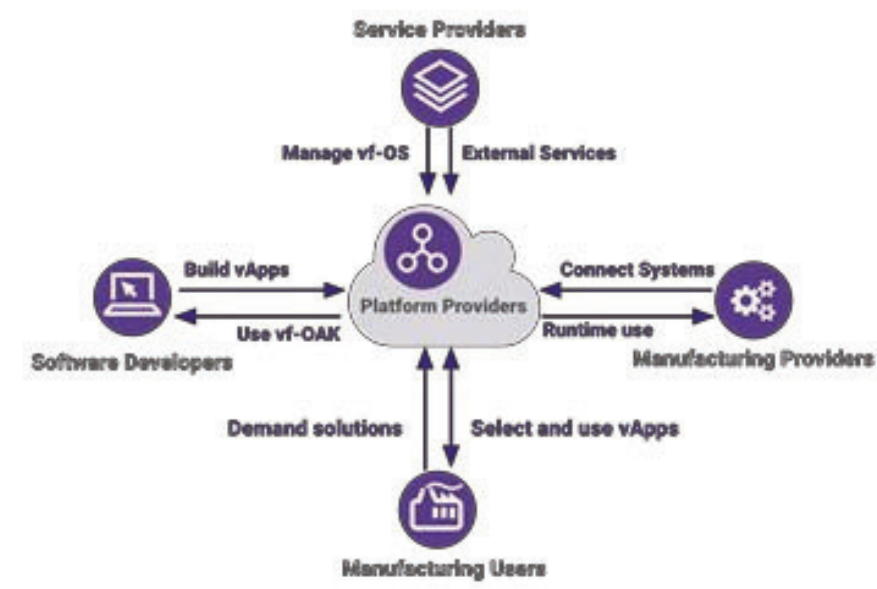

Fig. 1. Vf-OS Platform Concept.

Within vf-OS, manufacturing companies can browse the marketplace to find applications (vApps) that fit their specific needs. If they do not find any suitable vApp, they can request the development of a custom solution. Software Developers use the provided Development Kit (vf-OS Open Application Development Kit, or OAK in short) to build and upload vApps to the marketplace. IT Service Providers can manage Platform Services in the cloud and also extend the native vf-OS Platform functionalities by integrating external services. Finally, Manufacturing Providers who provide manufacturing and logistics equipment and services can connect their systems into the platform to provide new added value services to their offering through the vf-OS Platform.

Manufacturing Providers or Manufacturing Users who want to connect their systems, that is, either physical assets (eg devices) and virtual assets (eg legacy software), to the vf-OS platform need appropriate tooling, which should be very friendly for users in the manufacturing sector, easy to integrate and automate, secure by default and very flexible in order to support the plethora of available industrial communication protocols, legacy software interfaces or proprietary file formats available. 
The vf-OS IO Toolkit Generator is a design tool to connect factory assets to the vf-OS Platform, that is, both physical assets (eg devices) and virtual assets (eg legacy software) within the factory that has been designed with these requirements in mind. The vf-OS IO Toolkit Generator applies the principles of software scaffolding [4] to the development of interoperability components for digital manufacturing platforms. Basically, in software scaffolding, a generator is a software tool that automates the development of software code to create an application skeleton (or scaffold). Thus, the output of the vf-OS IO Toolkit Generator is a working vf-OS IO Component that interconnects the platform to the specific manufacturing asset it has been designed for. As described in this paper, this novel approach facilitates the automation of advanced security testing of the generated IO Components, through integrations with the vf-OS Platform Security Components. The vf-OS IO Toolkit Generator can also be extended to generate components for other platforms and even use to automatically generate the needed components to connect a factory the vf-OS Platform.

The rest of this paper is structured as follows. Section II describes the architecture and main frameworks used in the vfOS generator, including use case examples. Section III describes interactions between the IO Toolkit Generator and cybersecurity in vf-OS to provide a solid methodology to develop secure interoperability components. Section IV describes how the IO Toolkit Generator can be extended and integrated into other manufacturing platforms and environments. Finally, Section V includes some final conclusions and future work.

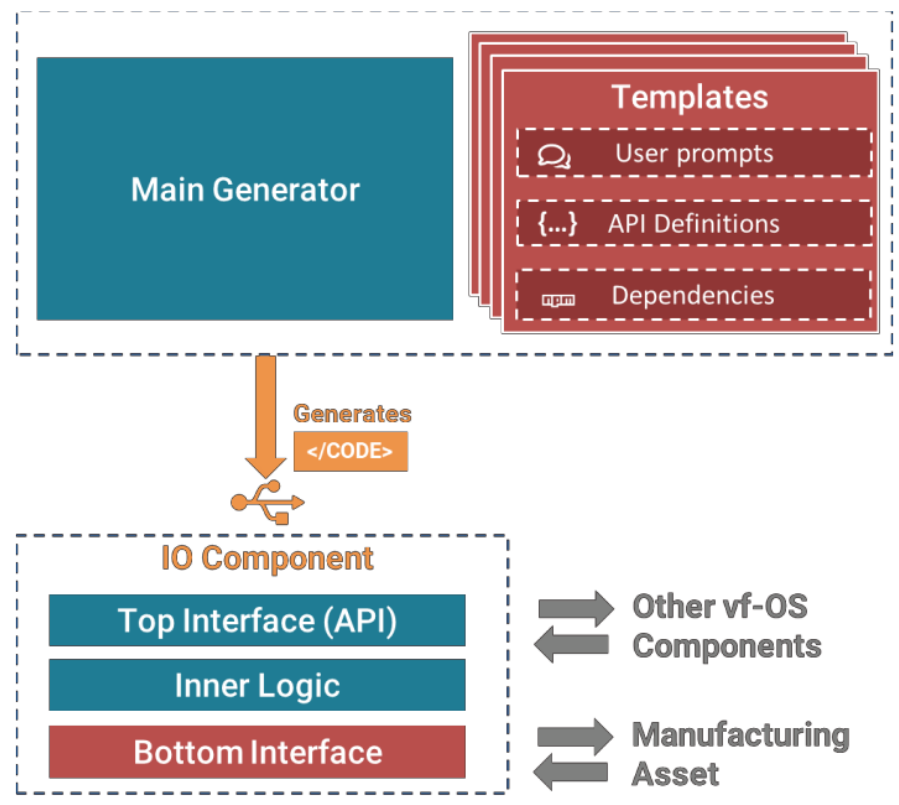

Fig. 2. Vf-OS IO Toolkit high level architecture.

\section{IO COMPONENTS SCAFFOLDING IN VF-OS}

\section{A. Vf-OS IO Toolkit Architecture}

Fig. 2 shows a high level description of the vf-OS IO Toolkit Generator and the generated output: a NodeJS [5] vfOS IO Component [6]. In the current release, the generator is based on the popular scaffolding framework for web applications Yeoman [7]. Yeoman provides a consistent workflow to automate software generation, starting from some basic input required to configure the desired output. The Yeoman workflow comprises three main tools: The Yo generator, the build system and the package manager.

The Yo generator is the tool that scaffolds the working copy of the new application, including the configuration files for the build tool and the package manager and therefore it is the main tool in the Yeoman framework. Yeoman generators can be published so that they can be used both by final users and within other generators, through a Yeoman feature called Composability.

Composability allows a generator to build upon other generators and reuse their functionality. Composability is used in vf-OS at early stages of the project to generate the first component prototypes using already available generators suitable for the project needs. In this sense, all vf-OS IO components are web applications that provide a RESTful Application Programming Interface (API) [8] to other vf-OS components. The vf-OS IO Toolkit Generator uses composability to build upon the Loopback [9] and Swagger [9][10] Yeoman Generators, so that a working web application is generated from an OpenAPI [11] specification of the REST contract of the component. Since the specification might change at this stage of the project, this allows to update the generated code just by changing the specification of the component. As described in Section IV, composability is also relevant to extend the generator so that it can be used to build interoperability components in other platforms.

The RESTful API, together with other core functionalities, comprises the Top Interface, which is the terminology used to refer to the interfaces and functionalities needed to interact with other components within the vf-OS Platform. The Top Interface is common to all vf-OS IO Components. Besides the Top Interface, an IO component instance has a Bottom Interface to interact with the specific factory asset it has been designed for and an Inner Logic, that is the internal business logic of the component.

From this high-level IO Component architecture, the vf-OS IO Toolkit Generator implements a templating framework where the main generator creates the code for the common functionalities to all components (basically the Top Interface and the Inner Logic) and each template controls the subgenerator producing the code which is specific to a particular IO Component. In vf-OS, the IO Toolkit provides a set of libraries to implement the Bottom Interface, referred to as the IO Toolkit SDK. Each template defines the dependencies needed to test and install the component, including the libraries within the IO Toolkit SDK that are needed to connect to a factory asset. Based on composability, templates can extend other templates to foster code reusability. Moreover, templates can request additional input from the user so that the generated output is configured according to their needs. Depending on the 
requested input, the generated code can either be an IO Component skeleton or a fully configured IO Component. The next section describes the basic structure of an IO Component Template.

\section{1) IO Components Templates}

The template for scaffolding an IO component consists of a JSON file containing the specification of the behaviour of the subgenerator (ie the template specification file, named data.js) and a files subfolder containing the files needed to write the generated code with the Bottom Interface implementation. The IO Toolkit defines a contract module that is used to interconnect the IO Component business logic to the Bottom interface implementation. The generator will generate the code that queues the asynchronous functions defined in this contract in the appropriate application callback stacks, so that they are called in response to events in the Top Interface. Thus, in order to develop a new template, it is only necessary to modify the contract module in the files folder to implement the Bottom Interface functionalities and add the required additional files to this folder and specify the required external dependencies the template specification file. For convenience, the contract modules for each IO Component Type (Device Drivers for components that connect to physical assets and API Connectors for components that connect to virtual assets) are defined in separate files. Fig. 3 shows the structure of the contract module for Device Drivers.

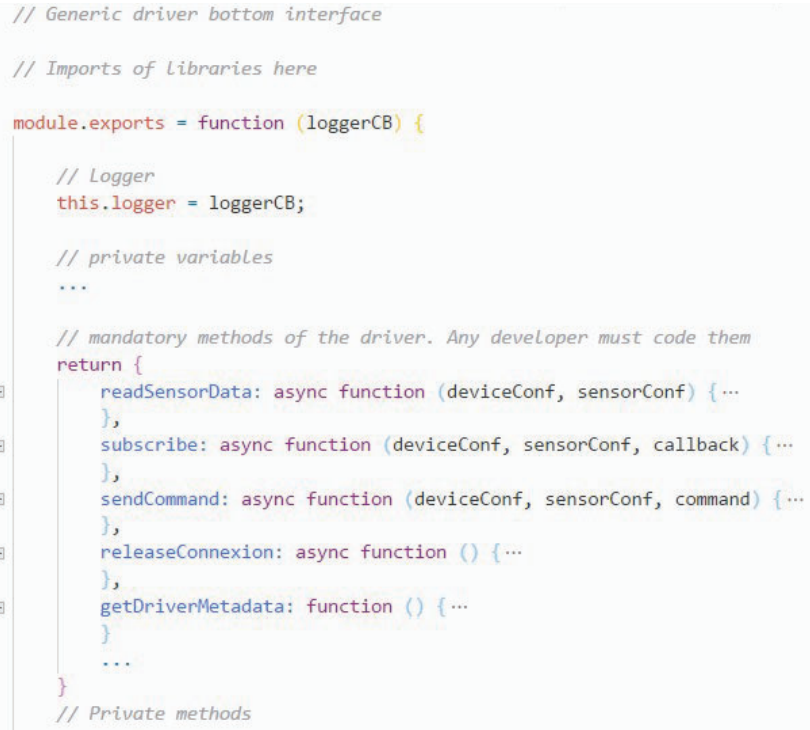

Fig. 3. Bottom Interface contract module for drivers.

Thus, the files subfolder contains the implementation of the Bottom Interface functionalities and the data.js file contains a set of JSON objects that specify declaratively the behaviour of the sub-generator. The main objects within this specification are:

Name: Name used to identify the template within the IO Toolkit Generator.

Description: A brief description of the generated code provided to the users.

Type: IO Component type (“driver” for Device Drivers and "connector" for API Connectors).
Package: Additional Information to write to the package file (Optional).

Swagger: Location in the files folder of the OpenAPI definition file describing additional (proprietary) models used by the component.

Files: The Files object controls the Yeoman files utilities [12] that copy files from the file subfolder of the template to the generated output. It consists of an Array of IO objects containing a source field with the location of the file structure to copy and a destination field with the location of the output file structure in the generated code where it will be copied.

Prompts: Array of questions to ask the user for additional information. Questions follow the Inquirer question object notation [13] and are used to control user interaction, adding new user prompts to the main generator to ask the user for further information as described in Section II.B.

Dependencies: Array of NPM [14] modules to add to the component dependencies to be processed by the package manager (Optional).

Fig. 4 shows an example of a template specification file for an OPC UA Driver template.

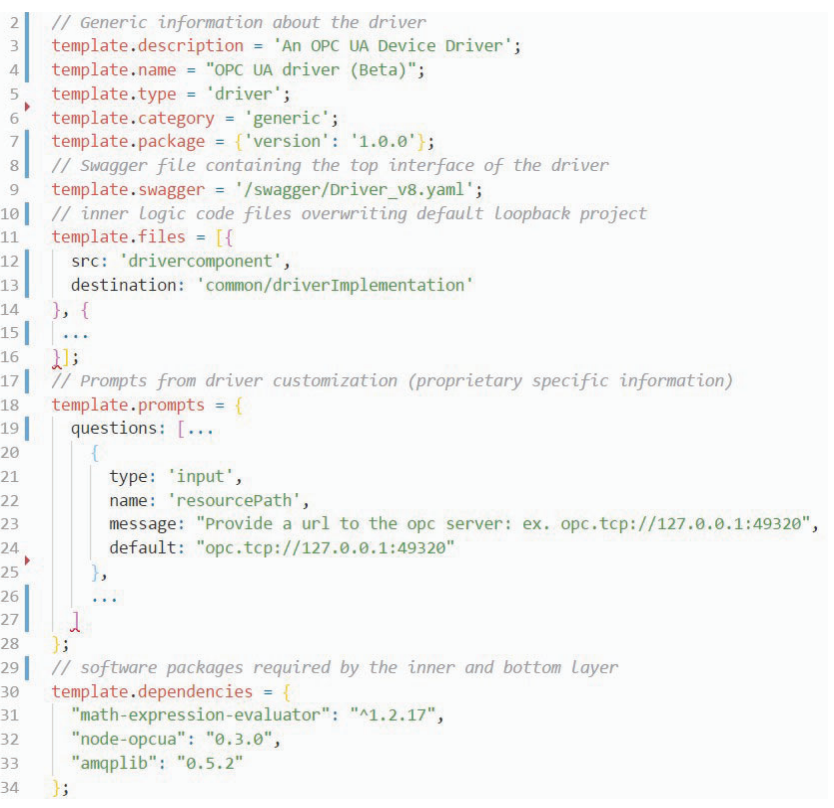

Fig. 4. Template specification file example.

This way, when developing a specific Bottom Interface with the contract module, the vf-OS IO Generator can assure the proper driver instantiation and usage by the inner logic and Top Interface, and in extension, the proper integration with other vf-OS Components like the Pub/Sub and Messaging IO component [6]. In this sense, getDriverMetadata, is a relevant method, as far as it returns a couple of arrays with the proprietary attributes that the generator must ask to get a properly configured driver according to the given protocol (e.g. namespace is a specific attribute in the OPC UA protocol [15], while topic is a specific (aka proprietary) attribute of sensors supporting the MQTT [16] protocol). This protocol specific information can be configured by the generator user either via 
command prompts during the design time or through the IO Component user interface during runtime as described in the next sections.

\section{B. Vf-OS IO Toolkit Generator User Interaction}

Yeoman provides different mechanisms to control the interaction with the user [17]. The default user interface is the terminal, but it is possible to integrate generators inside other graphical interfaces, as long as the provided user interface element abstractions - prompts, arguments and options - are used. The vf-OS IO Toolkit Generator implements user prompts to allow the user to select the IO Component type and template. As explained above, each template can provide a set of additional user prompts. The sub-generator will collect the input provided by the user and apply the provided configurations to the generated code. Options on the other hand are used to provide high level configuration parameters. The user interaction is explained in more detail through an example in the following subsections.

\section{1) User Prompts}

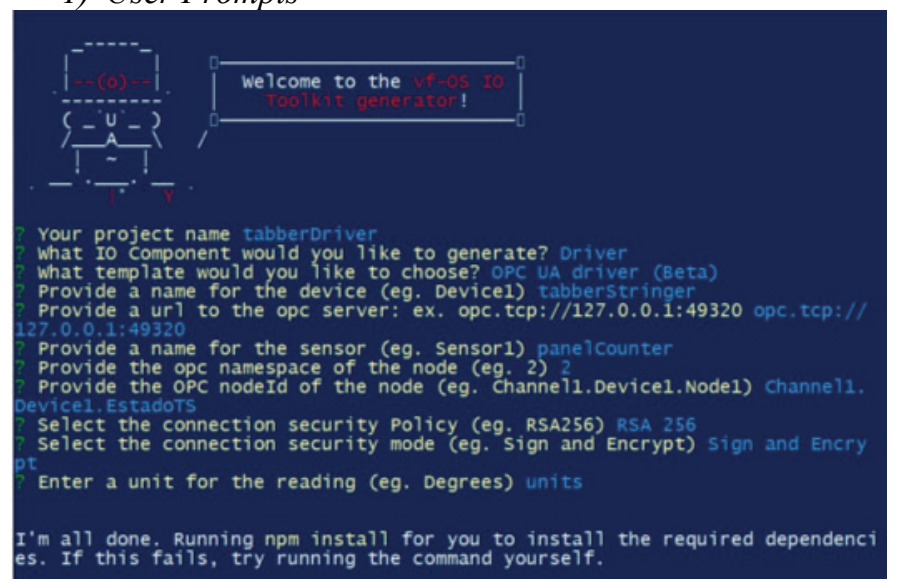

Fig. 5. Vf-OS IO Toolkit generator prompts.

As described above, user prompts are the main method to provide user input to apply the user preferences to the generated code. User prompts are command-line questions [13] that the user runs into when using the vf-OS IO Toolkit generator. As shown in the example in Fig. 5, the user first selects the name of the driver and the type of IO Component to be generated. Then, the generator shows the list of available IO Component templates for the selected type. When selecting one of them, the generator will go through the user prompts provided in the template specification file (see Fig. 4) and will collect the answers provided by the user. The sequence of prompts depends on the answers provided at each step. In the example below, the input provided will configure the connection of the driver to the machine and sensor with the specific proprietary parameters, everything without the user coding or editing any file.

\section{2) Options}

Options are a method to pass information to a generator directly from a command-line while invoking it. The current version of the vf-OS IO Toolkit Generator supports two options. The loopback option is used when the user wants to generate a self-contained loopback web application. If this option is not provided, the generator will provide the bottom

This work was supported in part by the European Commission under the

Grant Agreement 723710 interface implementation of the driver, and a technical user will need to deploy it in a convenient already-existing environment. Fig. 6 shows a command line invoking the vf-OS IO Toolkit Generator using both available options.

\section{yo vfos-io --loopback --force}

Fig. 6. Vf-OS IO Toolkit generator options.

The second option is the force argument, used to omit some questions that the generator asks when overwriting the template files in the output project.

\section{3) Post Generation User Interaction}

After running the vf-OS IO Toolkit Generator, a functional driver component is generated and can be installed and instantiated either in a local NodeJS runtime environment or in a Docker [18] container.

For the sake of flexibility and adaptability, any IO Component generated using the vf-OS IO Toolkit Generator provides a management User Interface (UI) that can be accessed through a web browser. Through this UI, Manufacturing and Logistic Users and Providers will get information about the connected assets and their configurations. The management UI supports querying and editing parameters, checking the current component data, or checking the status of the connection. Fig. 7 shows the management UI of a Device Driver.

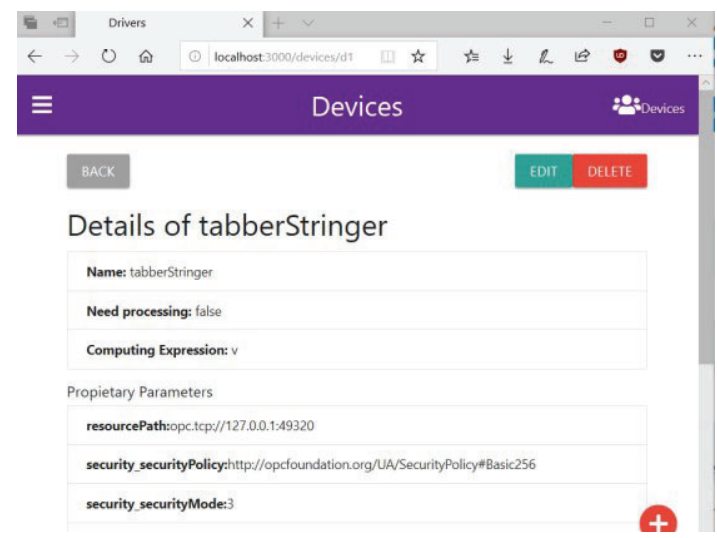

Fig. 7. Device Driver Management.

The information retrieved from the asset can be pushed into the vf-OS platform if the IO Component is configured accordingly. The protocol used for that purpose is AMQP [19], the communication protocol used by the vf-OS Pub/Sub and Messaging Component [6]. The vf-OS Pub/Sub and Messaging offers an API implemented on top of RabbitMQ [20], and provides mechanisms for the vApps to consume data from manufacturing assets with a fine grain control in terms of authorization as well as high flexibility to route messages in a secure way compared to MQTT.

\section{SOFTWARE SCAFFOLDING AND SECURITY}

The use of generators in the context of digital manufacturing platforms like vf-OS yields several benefits in the overall system security. As described below, The 
IO Toolkit Generator facilitates the integration of Static Application Security Testing (SAST) and Interactive Application Security Testing (IAST) [21] into the IO Component development workflow through the vf-OS Security Command Centre.

The vf-OS Security Command Centre [6] comprises the different platform components in charge of the security and privacy in vf-OS. Some relevant service components are the Identity Management Service and the Security Policy Management, which implements a Role Based Access Control (RBAC) [22] but also Attribute Based Access Control (ABAC) [23] when needed based on XACML [24] and FIWARE [25].

Another novel feature of the vf-OS Security and Privacy Concept is the Security Continuous Monitoring (SCM) Service, which is based on the Security Content Automation Protocol (SCAP) standard [26]. Basically, the SCM service facilitates the automation of vulnerability measurement and management, including policy compliance evaluation. Each vf-OS host implements an SCM agent that monitors the system status and is able to detect vulnerabilities registered in databases like the National Vulnerability Database (NVD) [27], according to the established security policies, but it also provides a confirmation of the needs to update and patch based on the presence of exploiting information in Open Source Exploiting Database Search Engines.

Section II explains how the IO Toolkit Generator prescribes the use of specific libraries to connect to factory assets, which are part of the IO Toolkit SDK. Templates use and extend these libraries to provide the developer with different options to generate a new IO component. Since the IO Toolkit SDK and Templates are open source code, it is possible to perform Static Analysis to identify vulnerabilities so that they are registered in the vulnerabilities database. Moreover, since both the IO Toolkit and the SCM are maintained by the vf-OS Project, it is possible to ensure that all the libraries and templates included are tested and verified and that a vulnerability report is available to the IO Toolkit Generator so that it can be included in the output. Thus, during the development of a new driver, the IO Toolkit Generator first creates code that has been scanned for security vulnerabilities, is adapted to comply with the required security profile through the generator and is accompanied by a vulnerability report. At the end, the generated source code is processed by the build system and package manager within the vf-OS development tools to create an IO Component which is secure by default.

Additionally, it is possible to integrate IAST into the component development workflow by testing the generated code using a Dynamic Application Security Testing (DAST) [21].

The vf-OS Security Agent uses the OpenAPI specification [11] to create a specific security profile which instruments the corresponding dynamic mechanism to test dynamically the application for finally generating a security report.

This development workflow, depicted in Fig. 8 is aligned with Secure by Design and Continuous Integration methodologies like SecDevOps [29].

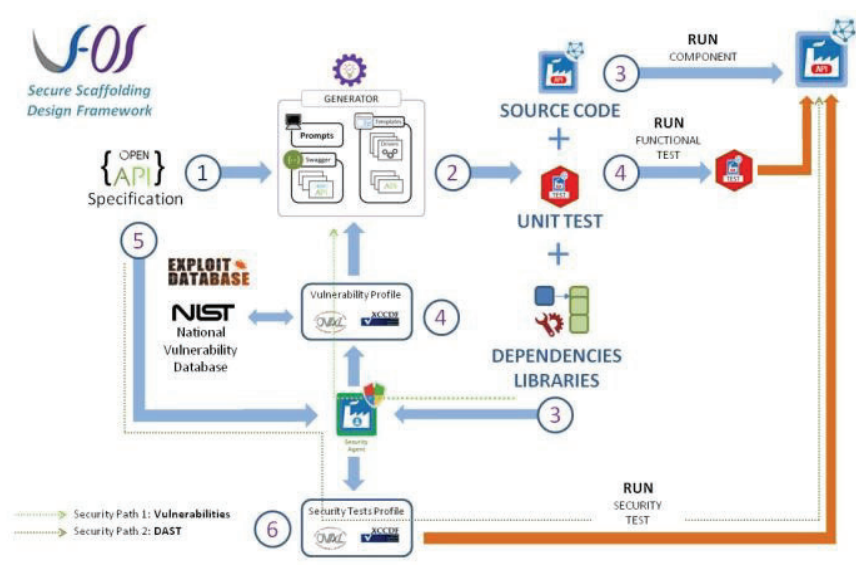

Fig. 8. Vf-OS IO Toolkit Generator and vf-OS Security Command Centre Integration.

\section{EXTENDING THE VF-OS IO TOOLKIT GENERATOR}

The vf-OS IO Toolkit Generator can be extended to generate interoperability components for other digital manufacturing platforms, for instance, prominent Open Source Platforms like FIWARE. As mentioned in Section II, the OpenAPI definition controls the REST API interface towards the Platform and the loopback option can be used to generate a web application that implements the REST interface with the Loopback framework. Similar options can be defined to extend the generator to develop interoperability components for other digital manufacturing platforms. For instance, a fiware option can be used to configure the generator to create a FIWARE IoT Agent [30]. If the user selects this option, the generator creates the code that implements the (Norhbound) interfaces towards the FIWARE Context Broker and IoT Agent Manager Enablers and connects it to the code provided by the template. As long as the abstraction of the manufacturing asset functionalities provided by the contract module can be used in the new platform, the templates can be reused and the required modifications to support a new digital manufacturing platform through an option are minimum.

Generators can be configured to be fully controlled by other systems instead of user interactions. This opens up new possibilities for automation and makes it possible to generate and instantiate on the fly ad-hoc components to interact with manufacturing assets. For instance, this can be used to develop functionalities to create interoperability components that adapt automatically to their environment, both towards the manufacturing platform where they are deployed and towards the manufacturing asset they need to interconnect.

\section{CONClusions}

This paper has presented the vf-OS IO Toolkit Generator, a software development tool that is used to generate interoperability components that connect manufacturing assets (both physical and virtual) to the vf-OS Platform. The vf-OS IO Toolkit applies an automated code generation technique, scaffolding, to the generation of interfaces to machines, legacy applications and file formats. This is a novel approach addressing interoperability in Cyber-Physical Systems. 
The use of automated code generation techniques makes it easier for the user to create and maintain components to connect their systems to the vf-OS Platform. This is especially important if cybersecurity is taken into consideration, because protecting software against cyber-threads is a challenging task. In this sense, this paper proposes integrations between the IO Toolkit Generator and vf-OS Security Components to integrate secure development techniques into the development workflow.

The IO Toolkit Generator is still under development and new functionalities will be implemented throughout the project. More IO Components are to be developed and tested in the different project pilots and the results will be fed back to the IO Toolkit Generator. Additionally, some possible lines of work are extending the generator to be used in other digital manufacturing platforms and develop integrations to completely automate the generation of interoperability components.

\section{ACKNOWLEDGMENTS}

The research leading to these results has received funding from the European Union H2020 Program under grant agreement No. 723710 "Virtual Factory Open Operating System" (vf-OS).

\section{REFERENCES}

[1] Kagermann, H., Helbig, J., Hellinger, A., Wahlster, W.: Recommendations for implement-ing the strategic initiative INDUSTRIE 4.0: Securing the future of German manufacturing industry; final report of the Industrie 4.0 Working Group. Forschungsunion (2013).

[2] Coopers, P. (2016). Industry 4.0: Building the digital enterprise. Key findings from 2016 Global Industry 4.0 Survey-Finland's perspective.

[3] vf-OS Project Homepage, http://www.vf-os.eu, last accessed 2018/07/23.

[4] Wintermeyer, S. (2018). Scaffolding and REST. In Learn Rails 5.2 (pp. 183-216). Apress, Berkeley, CA.

[5] Tilkov, S., \& Vinoski, S. (2010). Node, js: Using JavaScript to build high-performance network programs. IEEE Internet Computing, 14(6), 80-83.

[6] Vf-OS Architecture (I-ESA).

[7] Yeoman Homepage, http://yeoman.io, last accessed 2018/07/23.

[8] Wilde, E., \& Pautasso, C. (Eds.). (2011). REST: from research to practice. Springer Science \& Business Media.

[9] Loopback Homepage, https://loopback.io/, last accessed 2018/07/23.
[10] Team, S. (2014). Swagger restful api documentation specification 1.2. Techn. Ber. Technical report, Wordnik, 14-30.

[11] OpenAPI Homepage, https://swagger.io/specification/, last accessed 2018/07/23

[12] Yeoman File utilities, http://yeoman.io/authoring/file-system.html, last accessed 2018/07/28.

[13] Inquirer Homepage, https://github.com/SBoudrias/Inquirer.js, last accessed 2018/07/28.

[14] NPM Homepage, https://www.npmjs.com, last accessed 2018/07/28.

[15] Leitner, S. H., \& Mahnke, W. (2006). OPC UA-service-oriented architecture for industrial applications. ABB Corporate Research Center.

[16] Hunkeler, U., Truong, H. L., \& Stanford-Clark, A. (2008, January). MQTT-S-A publish/subscribe protocol for Wireless Sensor Networks. In Communication systems software and middleware and workshops, 2008. comsware 2008. 3rd international conference on (pp. 791-798). IEEE.

[17] Yeoman User Interactions, , http://yeoman.io/authoring/userinteractions.html, last accessed 2018/07/28.

[18] Merkel, D. (2014). Docker: lightweight linux containers for consistent development and deployment. Linux Journal, 2014(239), 2.

[19] Anderson, A., Nadalin, A., Parducci, B., Engovatov, D., Lockhart, H., Kudo, M., ... \& Moses, T. (2003). extensible access control markup language (xacml) version 1.0. OASIS.

[20] Videla, A., \& Williams, J. J. (2012). RabbitMQ in action: distributed messaging for everyone. Manning.

[21] Mendelev, K., Ragoler, I., Chess, B. V., Firestone, S. J., \& Kfir, Y. (2013). U.S. Patent Application No. 13/331,777.

[22] Ferraiolo, D., Cugini, J., \& Kuhn, D. R. (1995, December). Role-based access control (RBAC): Features and motivations. In Proceedings of 11th annual computer security application conference (pp. 241-48).

[23] Hu, V. C., Kuhn, D. R., Ferraiolo, D. F., \& Voas, J. (2015). Attributebased access control. Computer, 48(2), 85-88.

[24] Extensible Access Control Markup Language (XACML) Specification. Available Online: http://docs.oasis-open.org/xacml/3.0/xacml-3.0-corespec-cs-01-en.pdf, last accessed 2018/07/31.

[25] FIWARE Homepage, https://www.fiware.org/, last accessed 2018/07/31.

[26] The Technical Specification for the Security Content Automation Protocol (SCAP): SCAP version 1.3. Available Online, https://csrc.nist.gov/projects/security-content-automation-protocol/, last accessed 2018/07/31.

[27] National Vulnerability Database. Available Online, https://nvd.nist.gov/, last accessed 2018/07/31.

[28] Remote Exploits - Exploit Database. Available Online, www.exploitdb.com, last accessed 2018/07/31.

[29] Ur Rahman, A. A., \& Williams, L. (2016, April). Security practices in DevOps. In Proceedings of the Symposium and Bootcamp on the Science of Security (pp. 109-111). ACM.

[30] FIWARE IoT Stack. Available Online: https://catalogueserver.fiware.org/iot_stack, last accessed 2017/07/31. 